\title{
Hashimoto's Encephalopathy: A Diagnosis in Disguise, Case Report and Review of Literature
}

\author{
Lakshmi Kant Pathak $^{\mathrm{a}, \mathrm{c}}$, Vimala Vijayaraghavan ${ }^{\mathrm{b}}$
}

\begin{abstract}
While Hashimoto's encephalopathy (HE) is quite rare (there may only be several dozen diagnosed patients in the USA), it is also likely that there are many more undiagnosed sufferers. Because it is little known and its symptoms are primarily neurological, it is easy to misdiagnose or overlook and the symptoms frequently lead to mistaken neurological diagnoses. We report a case of an elderly female presenting with aphasia, cognitive decline, dysphagia and left hemiparesis as manifestation of this disorder. She failed to respond to high dose steroids and intravenous immunoglobulins which can be seen in rare subsets of patients. HE is a neuropsychiatric disorder of exclusion. It is important to identify this disease as most could be treated with steroids and immunosuppressants and therefore the term steroid-responsive encephalopathy associated with autoimmune thyroiditis (SREAT). Diagnosis is made in the first instance by excluding other toxic, metabolic and infectious causes of encephalopathy with neuroimaging and CSF examination. Response to treatment is quite variable despite most patients being steroid responsive. There are small subsets of patients who show poor response to steroids and immunoglobulins including this one and may require prolonged treatment to see any improvement.
\end{abstract}

Keywords: Hashimoto's encephalopathy; Thyroiditis; Autoimmune

\section{Introduction}

Hashimoto's encephalopathy (HE) is a rare neuroendocrine disorder associated with Hashimoto's thyroiditis. While HE is quite rare, it is also possible that there are many more undiagnosed cases. Because it is little known and its symptoms are primarily neurological, it is easy to misdiagnosis or overlook

Manuscript accepted for publication November 14, 2014

anniversity of North Dakota School of Medicine, Fargo, ND, USA

${ }^{\mathrm{b}}$ Caribbean Medical University School of Medicine, Curacao, Chicago Campus, IL, USA

${ }^{c}$ Corresponding Author: Lakshmi Kant Pathak, University of North Dakota School of Medicine, Fargo, ND 58122, USA. Email: drpathaklk@gmail.com

doi: http://dx.doi.org/10.14740/jmc1997w and the symptoms frequently lead to mistaken neurological diagnoses.

Treatment is usually high dose steroids and immunosuppressants, to which majority of the patients respond but there are occasionally rare cases unresponsive to this conventional treatment and pose difficulty.

If treatment is delayed due to misdiagnosis, it often becomes fatal and therefore physicians should have high degree of suspicion and awareness for this condition.

\section{Case Report}

The patient is an 82-year-old female with a history of hypertension, CAD, hyperlipidemia, and a recent history of a right frontal ischemic stroke with no motor deficit. The patient at the time had some aphasia; however, throughout the hospital stay, she improved, and she was discharged to home with outpatient physical therapy and occupational therapy.

The patient, as per daughters, was doing fairly well. She was independent enough to walk by herself and was able to communicate and hold up a conversation with her friends, but in the last 1 week, they noticed a rapid decline in her mentation. She was more confused. Her speech was clear; however, the context of speech was abnormal. She was very weak and requiring assistance to get her out of the bed. Her oral intake had reduced and she had poor sleep pattern with only few hours of sleep in last few days.

The patient had a follow-up in neurology clinic and underwent an MRI which did not show any new ischemic areas. It just showed evolutionary changes of her right frontal lobe ischemic stroke. She did not have fever, nausea, vomiting, abdominal pain, or diarrhea.

There was no new focal motor or sensory deficit. The patient had dysphasia and was disoriented. She had no seizure like activity or drowsiness. She was not on any new medications including sedatives or narcotics.

Physical examination revealed disorientation to time, place and person with poor attention. Interestingly, her naming/repetition/comprehension was all intact, though she did have some dysfluency of speech and slow mentation. She had no dysarthria.

Cranial nerves were intact and pupils were equal and reactive. 
Strength $5 / 5$ in proximal and distal upper and lower extremities bilaterally with normal tone and bulk. There were no abnormal movements and no pronator drift. Sensation intact to touch. Extensive workup was done to rule out any infectious, vascular, neoplastic or autoimmune cause.

\section{Pertinent lab work}

Thyroid peroxidase antibody titer was markedly positive at more than $10,000 \mathrm{IU} / \mathrm{mL}$ and thyroglobulin antibody at 21.5 $\mathrm{IU} / \mathrm{mL}$. TSH, free T4 and free T3 were all within normal values. Serum ammonia: 24. B12: normal. Lyme: nonreactive. RPR: nonreactive. Paraneoplastic panel: negative. ESR: 52. RF: < 15. ANA: negative. ANCA: negative. West Nile PCR: negative. HSV: negative. Fungal culture: negative. Viral culture: negative. Cytology (CSF): negative. Acid fast bacillus (CSF): negative. Bacterial stain (CSF): no organisms, culture negative. CSF electrophoresis: elevated gamma. No oligoclonal bands. EEG: abnormal. Background rhythms are diffusely slow and irregularly maintained, consistent with a diffuse encephalopathy, which may be toxic or metabolic, amongst other etiologies. Additionally, there are intermittent rhythmic intrusions of slow activity, more prominently noted over the right hemisphere, and specifically over the right frontal head region. These findings may be consistent with underlying structural or functional abnormality in that area, and also consistent with a diagnosis of encephalopathy. However, the focal and paroxysmal findings of this nature, although not frankly epileptiform, may raise concern for electrographic seizure.

With these findings, the patient was commenced on keppra with repeat EEG not showing any concern for seizure.

\section{Clinical course}

Patient symptomology, negative metabolic, infectious and immunological work up and presence of grossly positive thyroid antibodies were consistent with a diagnosis of Hashimoto's encephalopathy. The patient was treated with 7 days course of high dose solumedrol with no significant improvement unlike most cases of steroid-responsive thyroid encephalopathy. She was further treated with 5 days course of IV immunoglobulins which resulted in some improvement, although clearly not at baseline and continued to have fluctuating mental status.

She also developed progressive neurological deficit with left hemiparesis, dysphagia and diplopia. She needed a feeding tube for nutrition.

She was discharged to $\mathrm{NH}$ with high dose of steroids and follow-up in clinic.

\section{Discussion}

HE is a rare autoimmune disease associated with Hashimoto's thyroiditis. The first case of HE was described by Brain et al in 1966 [1]. The patient was a 48-year-old man with hypothyroidism, multiple episodes of encephalopathy, stroke-like symptoms and Hashimoto's thyroiditis confirmed by elevated anti-thyroid antibodies. It is recognized as a rare disease by the NIH Genetic and Rare Diseases Information Center. Since its better understanding and more research on the subject numerous cases are being reported by scientists around the world, it is suggested that this rare condition is likely to have been significantly undiagnosed in the past. Over 100 scientific articles on HE were published between 2000 and 2013.

It is a relapsing-remitting and sometimes progressive encephalopathy occurring in association with Hashimoto's thyroiditis. Initially it was referred as neuro-endocrine disorder but its endocrine basis is now widely disputed. It remains somewhat controversial disorder [1]. The prevalence has been estimated to be $2.1 / 100,000$ with female to male ratio of $4: 1$. The mean age of onset is 44 with $20 \%$ of cases presenting before the age of 18 years. Most reported cases occur during the patient's fifth decade of life.

The mechanism of pathogenesis is not known but it has been assumed to be an autoimmune disorder. Consistent with this hypothesis alpha-enolase has been identified as an autoantigen in the disease [2].

Very little is known about the pathophysiology of this disease. Most evidence suggests an auto-immune vasculitis or other immune-mediated process affecting the cerebral microvasculature. Post-mortem studies of some individuals have shown lymphocytic vasculitis of venules and veins in the brain stem and a diffuse gliosis involving gray matter more than white matter [2-4].

The relationship between Hashimoto's thyroiditis and HE is at best unclear. Some argue it as a spurious co-relation as high titers are common in general population [5].

Others debate that the relationship is just as seen among other autoimmune diseases in general [3]. Support for this comes from the fact that symptoms of HE do not co-relate with thyroid antibody titers or symptoms of thyroiditis. The fact is that like other autoimmune disorders this also responds to steroid therapy, and many propose to call it steroid-responsive encephalopathy associated with autoimmune thyroiditis (SREAT).

The clinical presentation is myriad and often confusing to the clinicians. Most often it presents as acute to sub-acute onset of confusion with change in consciousness [6,7]. Two patterns have been described, a stroke-like pattern with multiple, recurrent episodes of focal neurological deficit associated with cognitive dysfunction and alteration in consciousness [3]. The other pattern is less prominent and consists of progressive cognitive impairment with dementia, hallucination and somnolence [6]. Some cases can rapidly progress to coma.

The symptoms in adolescents, unlike adults, frequently include seizures, confusion and hallucinations. A drop in school performance is also a common symptom, along with progressive cognitive decline.

The clinical patterns can overlap. The long-term course of illness may be self-limited, relapsing-remitting, or progressive.

The most common laboratory findings are elevated LFTs and thyroid antibodies found in more than $50 \%$ of cases. Thyroid hormone abnormalities are common including hypo- and 
hyperthyroidism but around $25 \%$ patients remain in euthyroid.

Thyroid antibodies, both anti-thyroid peroxidase antibodies (anti-TPO, anti-thyroid microsomal antibodies, and anti$\mathrm{M}$ ) and anti-thyroglobulin antibodies (anti-Tg), in the disease are elevated but their levels do not correlate with the severity.

A single photon emission CT shows focal and global hypo-perfusion in majority $(75 \%)$ of patients.

EEG studies, while almost always abnormal (98\% cases), are usually non-diagnostic. The most common findings are diffuse or generalized slowing or frontal intermittent rhythmic delta activity [6]. Prominent triphasic waves, focal slowing, epileptiform abnormalities, photo paroxysmal and photo myogenic responses may also be seen. These finding are nonspecific and may be seen in toxic, metabolic and post anoxic encephalopathy.

Due to its variety of clinical symptomology, this condition may be difficult to diagnose at initial presentation and may mimic stroke, rapidly progressive dementia, Creutzfeldt-Jakob disease, para-neoplastic or viral encephalitis. This poses a diagnostic challenge and requires extensive workup to rule out toxic, metabolic, vascular and infective causes. It is often also called investigation negative encephalopathy.

\section{Treatment}

Most patients respond to high dose steroid therapy and therefore it is also called SREAT.

Initial treatment is with oral prednisone or high dose IV solumedrol for 7 days. Thyroid hormone treatment is also needed in patients with concurrent thyroid disorder. Treatment with corticosteroids is almost always successful but there are rare subsets of patients who fail to respond to initial first-line treatment as this particular case. This led to many alternative therapies including immunosuppressants like azathioprine, methotrexate and cyclophosphamide which may be affective $[7,8]$, along with immunomodulation with periodic IV immunoglobulins and plasma exchanges [7,9].

None of this treatment has been studied in controlled trials and therefore there are no clear guidelines for their use.

Seizures if occur should be treated with ant-convulsants. Duration of treatment depends on response and may vary from 2 to 20 years. Relapse is common after discontinuation of ini- tial treatment. Left untreated it is a fatal disease.

In summary, Hashimoto's encephalitis is a disorder of presumed autoimmune origin characterized by high titers of thyroid antibodies. It has a relapsing and remitting course characterized with various neuropsychiatric symptoms.

More research is needed to determine the association between the clinical manifestation, thyroid disease and autoimmune origin. Until then it is fair to include it in nonvasculitic, autoimmune inflammatory meningoencephalitis.

\section{References}

1. Brain L, Jellinek EH, Ball K. Hashimoto's disease and encephalopathy. Lancet. 1966;2(7462):512-514.

2. Yoneda M, Fujii A, Ito A, Yokoyama H, Nakagawa H, Kuriyama M. High prevalence of serum autoantibodies against the amino terminal of alpha-enolase in Hashimoto's encephalopathy. J Neuroimmunol. 2007;185(12):195-200.

3. Chong JY, Rowland LP, Utiger RD. Hashimoto encephalopathy: syndrome or myth? Arch Neurol. 2003;60(2):164-171.

4. Duffey P, Yee S, Reid IN, Bridges LR. Hashimoto's encephalopathy: postmortem findings after fatal status epilepticus. Neurology. 2003;61(8):1124-1126.

5. Sunil GS, Mariash CN. Hashimoto's encephalitis. J Clin Endocrinol Metab. 2001;86(2):947.

6. Kothbauer-Margreiter I, Sturzenegger M, Komor J, Baumgartner R, Hess CW. Encephalopathy associated with Hashimoto thyroiditis: diagnosis and treatment. J Neurol. 1996;243(8):585-593.

7. Shaw PJ, Walls TJ, Newman PK, Cleland PG, Cartlidge NE. Hashimoto's encephalopathy: a steroid-responsive disorder associated with high anti-thyroid antibody titers-report of 5 cases. Neurology. 1991;41(2 (Pt 1)):228-233.

8. Chaudhuri A, Behan PO. The clinical spectrum, diagnosis, pathogenesis and treatment of Hashimoto's encephalopathy (recurrent acute disseminated encephalomyelitis). Curr Med Chem. 2003;10(19):1945-1953.

9. Jacob S, Rajabally YA. Hashimoto's encephalopathy: steroid resistance and response to intravenous immunoglobulins. J Neurol Neurosurg Psychiatry. 2005;76(3):455-456. 